\title{
Dispositivos didácticos para la enseñanza- aprendizaje del lenguaje escrito en la universidad: algunos lineamientos para su elaboración
}

\author{
Maria Ignacia Dorronzoro* \\ Maria Fabiana Luchetti**
}

\begin{abstract}
Resumo
En este artículo, nos proponemos presentar una serie de lineamientos teórico-metodológicos para el diseño de "dispositivos didácticos" destinados a la enseñanza-aprendizaje de contenidos de asignaturas de carreras humanísticas. Esos lineamientos, elaborados en el marco de un "Programa de Acompañamiento a docentes de la carrera de Ciencias de la Educación de la Universidad Nacional de Luján" (provincia de Buenos Aires, Argentina), se inscriben en la perspectiva sociocultural vigotskiana $\mathrm{y}$, desde ese enfoque, procuran poner de manifiesto la articulación de los contenidos disciplinares y las formas del discurso a través de las cuales se llevan a cabo y se comunican los razonamientos propios de cada campo del saber.
\end{abstract}

Palabras clave: Dispositivos didácticos. Conocimiento disciplinar. Lenguaje escrito. Universidad.

Recebido em:28/04/2017

Aceito em: 12/09/2017

\footnotetext{
* Universidad Nacional de Lujan, Departamento de Educación, Profesora Adjunta de Lectura y escritura académicas.

** Universidad Nacional de Lujan. Profesora auxiliar de lectura y escritura académicas.
} 


\section{Introducción}

En la actualidad, numerosos estudios abordan la temática de la lectura y la escritura académicas desde distintos enfoques. Por nuestra parte, a partir de los principios de la perspectiva sociocultural (VIGOTSKI, 1977, 1978; WERTSCH, 1988; KOZULIN, 2000), entendemos que se trata de prácticas sociales y que, como tales, varían en función de los contextos en los cuales se realizan. Es decir que no se trata de "técnicas universales" que se aprenderían de una vez y para siempre y luego se aplicarían a cualquier situación o contenido, sino que ellas se modifican de acuerdo con la actividad concreta del contexto en el que se llevan a cabo.

La educación superior debe ser comprendida, pues, en ese marco, como uno de esos contextos, caracterizado por la actividad de producción y comunicación de conocimientos en distintos campos disciplinares. Ese supuesto implica, para nosotras, la necesidad de que la universidad asuma la enseñanza de las particularidades de la producción e interpretación de los textos que circulan en los distintos campos del saber del contexto académico. Pero, además, creemos que la enseñanza de esas prácticas del lenguaje debe llevarse a cabo de manera articulada con los propósitos y contenidos con los que ellas se articulan. En efecto, desde esa perspectiva teórica, se cuestiona el tratamiento aislado de estrategias generales de comprensión lectora y de producción de textos y se remarca "la centralidad del contenido específico en el abordaje de los textos" (LERNER; AISEMBERG; ESPINOZA, 2011, p. 530).

En esa postura teórica, el trabajo del docente deviene esencial para que el estudiante logre desarrollar prácticas de lectura y escritura apropiadas para la construcción y la transmisión de conocimientos dentro del ámbito académico. En ese sentido, desde el área de Prácticas Académicas del Lenguaje de la División de Pedagogía Universitaria y Capacitación Docente de la UNLu se viene llevando a cabo, desde hace algunos años, una línea de acción que hemos denominado "Programa de Acompañamiento a docentes de la carrera de Ciencias de la Educación". Con él, se pretende generar un espacio para la reflexión compartida y el diseño de actividades que consideren la relación entre los contenidos disciplinares y las formas del discurso a través de las cuales se llevan a cabo y se comunican los razonamientos propios de cada campo del saber. 
En este artículo, nos proponemos presentar una serie de lineamientos u orientaciones teórico-metodológicas - construidos en el marco del mencionado programa -, para el diseño de "dispositivos didácticos" en los que se ponga de manifiesto la articulación contenido-lenguaje. Con ese propósito, entonces, en primer lugar, nos detendremos en algunas cuestiones teóricas que enmarcan la propuesta y luego pasaremos a la descripción de los lineamientos que fueron elaborados a la luz de la perspectiva sociocultural y como resultado de la experiencia realizada en el acompañamiento a los docentes.

\section{Algunas cuestiones teóricas}

\subsection{Relaciones entre contenido y lenguaje}

Así entonces, como acabamos de anunciar, es en el marco de ese programa donde fueron tomando forma (y aún lo siguen haciendo), a partir de los intercambios con los docentes, los lineamientos teórico-metodológicos que presentaremos en este artículo como fundamento para el diseño de dispositivos didácticos en los que se evidencie que el lenguaje es una parte constitutiva de los contenidos disciplinares. En ese sentido, recordemos que, a partir de la perspectiva vigotskiana, se sostiene que los significados de palabras - base de la comunicación humana - contienen al lenguaje y al pensamiento interrelacionados. En otras palabras, para comunicar no se puede prescindir ni de los signos, ni del reflejo generalizado de la realidad, esencia del significado que no se refiere a objetos aislados, sino a grupos o clases de objetos y relaciones (RIVIÈRE, 2002).

Tomando como fundamento esos supuestos, entendemos que los saberes disciplinares no tienen una existencia abstracta, independiente de sus formas verbales y de las prácticas de producción y recepción que los ponen en funcionamiento. Al contrario, desde esa perspectiva, el conocimiento es concebido como un hecho cultural y discursivo, una construcción social que "no forma parte de la naturaleza, sino que es la interpretación de ésta que ha realizado la comunidad humana, con el instrumento racional del lenguaje." (CASSANY, 2000, p. 74).

Por lo tanto, en ese marco, toda disciplina es concebida como una actividad social, como una construcción llevada a cabo por medio de la comunicación 
instaurada por el lenguaje que, lejos de ser entendido como una herramienta que "refleja" la realidad, se presenta como un instrumento de mediación, desarrollado culturalmente, que se incorpora a la acción humana dándole forma y controlándola a través de su doble función comunicativa y representativa. Así, se entiende que el discurso no es una vía por la que se transmiten mensajes, sino que, en tanto instrumento de mediación, se considera una actividad en la que se genera significado (CUBERO PÉREZ et al, 2008). En otros términos, el lenguaje no es concebido, aquí, como una simple representación del pensamiento, sino como un factor que fundamentalmente lo configura y lo define (WERTSCH, 1993).

Por lo tanto, aprender los conocimientos de cada disciplina es, para nosotras, aprender a emplear los modos de discurso que le son propios y constitutivos (CUBERO PÉREZ et al, 2007). Efectivamente, dado que las palabras no remiten a las cosas, sino a un universo de discursos, los estudiantes nunca se confrontan con un saber puramente ideal, sino con un saber instituido verbalmente. Es decir, nunca se enfrentan al saber en sí mismo, sino a los discursos sobre éste. Esa "institución" verbal estructura de manera determinante la apropiación del "contenido de saber" por lo que resulta imposible, para nosotras, disociar los contenidos disciplinares de las prácticas del lenguaje que los "instituyen".

Así, en ese marco teórico, las disciplinas dejan de representar sólo una serie de conocimientos codificados, y pasan a incluir, como elementos constitutivos, determinadas modalidades de pensamiento mediadas por formas propias de funcionamiento verbal, plasmadas en las distintas textualizaciones del saber que constituyen los textos académicos, tanto orales como escritos. Por lo tanto, la apropiación de los conocimientos disciplinares implica no sólo el dominio de un patrón temático de términos conceptuales, sino, también, el manejo satisfactorio de los modos característicos de plantear los problemas, de razonar y establecer relaciones, de representar la realidad; en definitiva, "de crear y expresar el conocimiento" (CUBERO PÉREZ et al, 2007, p. 12) dentro del campo del saber en cuestión.

Desde ese punto de vista, entonces, las prácticas del conocimiento y las prácticas discursivas son inseparables, lo que implica que la apropiación de conceptos disciplinares no puede llevarse a cabo independientemente del lenguaje. Así concebido, ese complejo proceso supone, necesariamente, para nosotras, un trabajo del docente orientado a que los estudiantes aprehendan no solo un contenido sino, sobre todo, el modo en que éste se construye en el discurso a partir de los usos del lenguaje propios del campo disciplinar. 


\subsection{Nuestra concepción de los dispositivos}

Dado que, como hemos planteado, nuestro propósito es presentar una serie de lineamientos teórico-metodológicos que sustenten el diseño de "dispositivos didácticos" - en ese caso, propuestas de enseñanza - en los que se ponga de manifiesto la articulación contenido-lenguaje, resulta necesario explicitar nuestra concepción de los dispositivos.

Así entonces, en términos generales, es posible decir que un dispositivo es "un espacio de interacción social y de cooperación compuesto de intenciones y materiales, que posee [además] un funcionamiento simbólico al igual que sus propios modos de interacción.” (MORALES; LENOIR; JEAN, 2012, p. 119). Son constructos a través de los cuales los enseñantes procuran "prever y balizar el recorrido de formación que proponen a sus estudiantes, bajo la influencia de sus elecciones didácticas y pedagógicas." (WEISSER, 2010, p. 292). ${ }^{1}$ Desde el punto de vista de su naturaleza, es necesario señalar que los dispositivos no se limitan sólo a objetos físicos, sino que, inclusive, pueden ser construcciones simbólicas. Es precisamente en ese tipo en el que nos centraremos en este trabajo.

Ahora bien, en ese marco general y desde la perspectiva del interaccionismo sociocultural en la que nos sustentamos, los dispositivos constituyen "recursos de mediación que organizan relaciones funcionales entre seres humanos, instrumentos, intenciones y acciones." (MORALES; LENOIR; JEAN, 2012, p.119). De ese modo, y siguiendo a esos mismos autores, el concepto de dispositivo aparece estrechamente imbricado con los de mediación y de acción, "entendiendo el primero como un espacio potencial en el cual es posible construir el saber, y el segundo, el de acción, en términos generales, como actos que son intencionales" (PETIT, 1990 citado por MORALES; LENOIR; JEAN, 2012, p. 120). Desde ese punto de vista, los dispositivos que proponemos elaborar, a partir de los lineamientos que hemos construido, se plantean como una propuesta didáctica,

resultado, virtual, de un trabajo de ingeniería que prevé las herramientas semióticas o instrumentales (VYGOTSKI, 1930/1985; MERCIER, 1998) que se pondrán a disposición del sujeto para que su relación con el mundo, se constituya en fuente de aprendizaje. ${ }^{2}$ (WEISSER, 2010, p. 292). 
Cabe señalar que, en nuestro caso, esa relación con el mundo está mediatizada por los contenidos disciplinares en articulación con el lenguaje.

\section{Lineamientos teórico-metodológicos propuestos para el diseño de los dispositivos didácticos}

Pasemos ahora a nuestra propuesta. Recordemos que nuestra intención es plantear algunas orientaciones teórico-metodológicas que permitan el diseño de dispositivos didácticos destinados a la enseñanza de contenidos pertenecientes al campo de las ciencias humanas y en los que, como se anticipó en el apartado anterior, se ponga de manifiesto su articulación con las formas del lenguaje que les son constitutivas. Por otra parte, es preciso advertir que esos lineamientos presentan distinto grado de generalidad y están inscriptos en un continuum en el que se considera como punto de partida la determinación ejercida por el contexto en los procesos de enseñanza-aprendizaje. De esa determinación, se desprenderán luego las demás orientaciones referidas a aspectos concretos que, a nuestro juicio, se deben considerar en el diseño de las intervenciones.

\subsection{El primer lineamiento}

El primer lineamiento de carácter general está dado por el condicionamiento que el contexto universitario ejerce sobre el proceso de formación y, específicamente, por la necesaria inscripción de ese proceso en el marco de una comunidad disciplinar, expresión del contexto antes mencionado.

Efectivamente, a partir de los principios de la perspectiva sociocultural, nuestra concepción del contexto supera la simple situación comunicativa y remite a una realidad mucho más vasta que es la esfera de actividad humana en la cual el lenguaje surge y que, simultáneamente es construida, conformada por ese lenguaje. Así, en ese marco, son las distintas actividades humanas las que delimitan los diferentes contextos con los que se articula el lenguaje, es decir que, los grupos que comparten determinadas actividades también comparten necesariamente ciertas modalidades peculiares de usos del lenguaje. De esa forma, las prácticas de lectura y escritura llevadas a cabo por los estudiantes universitarios deben ser 
entendidas insoslayablemente en función de la esfera de actividad, del contexto en el cual se inscriben, que es la actividad académico-institucional.

Ahora bien, la actividad académica es muy amplia y aunque se la puede caracterizar a partir de una misma finalidad, la de construir conocimientos, es necesario admitir que esa construcción no se hace de la misma manera en todas las disciplinas. Consecuentemente con ello, una caracterización del contexto de la educación superior debe considerar las diferentes comunidades que Olson (1998) denomina "comunidades textuales" y que nosotros llamaremos discursivo -disciplinares.

La cultura escrita es una condición cognitiva y social; al leer y escribir textos se participa de una "comunicad textual", un grupo de lectores (y autores y oyentes) que comparten un modo de leer e interpretar un corpus de textos. Convertirse en letrado en determinado campo es aprender a compartir un "paradigma". Kuhn (1962) propuso esta noción para describir una comunidad científica que comparte un conjunto de textos, de interpretaciones y de creencias como lo que plantea un problema para ulteriores indagaciones y análisis. Para ser letrado no basta con conocer las palabras; debe aprenderse cómo participar en el discurso de alguna comunidad textual. Y eso implica conocer qué textos son importantes, cómo deben leerse e interpretarse y cómo deben aplicarse en el habla y en la acción. (OLSON, 1998, p. 301).

Por nuestra parte, y tomando como punto de partida la definición de Olson, concebimos las comunidades discursivo-disciplinares como ámbitos en los que se lleva a cabo una serie de actividades tendientes a construir conocimientos, mediante la comunicación instaurada por modalidades particulares del lenguaje, a partir de determinados géneros desarrollados a lo largo de la historia de la disciplina de modo interrelacionado con el entrono físico y cultural (CASSANY, 2008). Esos usos particulares del lenguaje destinados a construir significados complejos y compartidos por los miembros de la comunidad, suponen un estilo propio (estrategias temáticas y retóricas), sus propios formatos de razonamiento, formas especiales de comunicar su contenido, ya sea hablado o escrito, y un uso interconectado particular de los términos específicos. La denominación de estas esferas de actividad como comunidades discursivo-disciplinares obedece pues, a nuestra concepción de las disciplinas como actividades sociales, y del papel sustancial del lenguaje en su constitución, tal como lo señalamos anteriormente.

Así entonces, cada comunidad discursiva construye y utiliza los discursos con 
distintos fines, por lo tanto, la comunicación en el interior de cada una de ellas no se limita a la transmisión de saberes disciplinares, sino que implica, sobre todo, un conjunto de significados lingüísticos, pragmáticos, retóricos, contextuales y sociocognitivos que los interlocutores deben compartir para poder comprender los discursos, actualizar el contenido y negociar su incorporación a la comunidad. De esa forma, los procesos de aprendizaje que tienen lugar en el marco de una comunidad disciplinar no pueden desconocer esas particularidades del contexto en el que se llevan a cabo. En ese sentido creemos, junto con Nussbaum y Tusón (1996) que

[...] aprender significa apropiarse paulatinamente de las formas de hablar y escribir (también de saber hacer y de saber decir lo que se hace) sobre el objeto de aprendizaje en cuestión, y hacerlo en la manera acostumbrada en esa parcela del saber; significa, por tanto, apropiarse del discurso específico que se reconoce como propio de esa disciplina. (NUSSBAUM Y TUSÓN, 1996, p. 5).

Desde ese punto de vista, el concepto de comunidad discursivo-disciplinar permite pensar el rol del discurso en la construcción de los saberes disciplinares y pone de manifiesto la especificidad que las prácticas del lenguaje revisten en el contexto universitario, haciendo posible "tomar distancia de las explicaciones clásicas de las dificultades de los estudiantes por supuestas carencias cognitivas o incompetencias vinculadas con el lenguaje" (DELCAMBRE, 2013, p. 179). En ese sentido, entonces, desde nuestra perspectiva, la enseñanza de las prácticas de lectura y escritura académicas no puede llevarse a cabo desde una intención remedial en relación con las carencias que se han producido en la escuela, sino a partir de un interés por la formación integral del estudiante como miembro de una comunidad disciplinar, es decir desde el compromiso por acompañarlos en su entrada a un mundo científico que tiene lenguajes propios, textos propios, y que le exige operaciones intelectuales complejas e inéditas para ellos. Tal como sostiene Calsamiglia (2000), creemos que

el acceso a este particular uso del lenguaje requiere una enseñanza sistemática que debe ser ofrecida en la formación superior. Se trata de la adquisición de una capacidad cognitivo-lingüística muy específica, alejada de la comprensión y del uso común de la lengua del hablante medio, tanto por su abstracción como por la combinación de lenguajes formales. Las disciplinas científicas se expresan hoy en un lenguaje desconocido, hermético y difícil para quien no es especialista. (CALSAMIGLIA, 2000, p. 4). 
La consideración de las dimensiones contextuales de la lectura y la escritura, implica, para nosotras, un abordaje de estas prácticas que considere ante todo su "situación" en un contexto preciso, en una determinada comunidad disciplinar. Un abordaje que, en ese caso, debe estar basado en la naturaleza primordialmente verbal de los modos de conocer dentro de cada disciplina.

Ahora bien, ¿cómo se ve reflejado ese condicionamiento de la comunidad disciplinar en el diseño de un dispositivo didáctico destinado a la enseñanzaaprendizaje de un determinado contenido? En función de lo hasta aquí expresado, creemos que ese diseño debe estar orientado a poner de manifiesto el fundamento mismo del concepto de comunidad disciplinar: la articulación entre contenido y lenguaje, la que se materializa, para nosotras, en las operaciones discursivocognitivas mediante las cuales se construyen los contenidos en los textos que se proponen como soporte del proceso de enseñanza-aprendizaje.

Así entonces, considerar ese condicionamiento de la comunidad disciplinar, implica trabajar didácticamente sobre la lógica de construcción del conocimiento en ese campo del saber, lógica que se ve plasmada en las particularidades que procedimientos tales como definir, fundamentar, explicar, adoptan en cada disciplina. Esas operaciones, que constituirían lo que Zemelman (2001) denomina la "lógica de construcción" de los razonamientos (ZEMELMAN, 2001, p. 13), permitirán, desde nuestro punto de vista, inscribir el contenido en una coherencia nocional o conceptual, y mostrarán al estudiante que el contenido es una construcción realizada mediante un uso específico del lenguaje. Es en ese sentido, que el trabajo con esas operaciones pondría de manifiesto la lógica de construcción de los saberes en función de pautas cognitivas y epistemológicas de la comunidad disciplinar (BAUTIER, 2009). En efecto, ellas constituyen las maneras convencionales de relacionar ideas y conceptos propios de un campo del saber, formas particulares de crear y expresar el conocimiento, "modos característicos de plantear los problemas, modos particulares de razonar y establecer relaciones, modos particulares de representar la realidad." (CUBERO PÉREZ et al, 2007, p. 12).

El dispositivo deberá, entonces, no solo procurar que el estudiante se apropie de un nuevo concepto, sino, también, de nuevas formas de comprender y explicar la realidad plasmadas en esas operaciones. En esa misma línea, se debe destacar que, tal como señala Casco (2014), esos procedimientos no suelen ser objeto de enseñanza en la universidad argentina, sino que, con frecuencia, la expectativa de los docentes es que los estudiantes los realicen natural y espontáneamente. De hecho, en muchos casos se espera que sea el estudiante quien descubra, de manera 
independiente y a través de una exposición frecuente a textos disciplinares, las formas específicas de usar el lenguaje para construir los conocimientos dentro del campo disciplinar.

Por el contrario, desde nuestra perspectiva, planteamos la necesidad de que los dispositivos didácticos pongan de manifiesto que cada disciplina académica posee su propio método para organizar el conocimiento en función de determinadas operaciones, y que inscriban la enseñanza de todo contenido en ese marco. Creemos, entonces, junto con Castelló (2009), que

donde más dificultades encuentran los alumnos es en el descubrimiento de las formas de organizar la información que promueven estos textos [académicos], precisamente porque la manera en que se ordenan y organizan las ideas en una discusión científica o en una monografía tiene mucho que ver con aquellos aspectos del conocimiento que se consideran más relevantes y útiles en cada disciplina, en un determinado momento histórico. (CASTELLÓ, 2009, p. 12).

Ahora bien, para cerrar este apartado, creemos conveniente ilustrar esas operaciones que materializan la articulación entre contenido y lenguaje. Así, por ejemplo, Castelló (2009) alude a la introducción de un texto que pretende presentar el resultado de una revisión bibliográfica. En ese caso, según la autora,

se puede optar por estructurar y organizar la información siguiendo criterios cronológicos o por el contrario, se puede optar por el criterio de evolución teórica; es posible también primar aquella información que en la actualidad sea más relevante o tal vez más compleja; o aún se puede organizar la información en función de los intereses y objetivos del que escribe. Los diferentes ejes estructuradores constituyen formas alternativas de organizar la información que pueden ser opcionales o preceptivas en según qué disciplinas y, en todos los casos, habrá opciones mejor valoradas que otras en cada comunidad científica que nunca resultan obvias para el estudiante. Algo parecido sucede con el nivel de subjetividad que se espera del autor en un ensayo o con la cantidad de reflexiones personales que se supone que debe incluir un informe. Las respuestas son específicas de cada disciplina e incluso de cada situación comunicativa. (CASTELLÓ, 2009, p. 12).

Otro ejemplo de esas operaciones del lenguaje, en ese caso, propia del campo de las ciencias humanas, es la definición y caracterización de un objeto de estudio 
a partir de su diferenciación de otro que se supone más cercano al estudiante. Efectivamente, como se sabe, esas operaciones implican el señalamiento y la explicación de las características de un objeto, hecho o relación. Ahora bien, en muchos textos de este campo del saber, el procedimiento se realiza tomando como punto de apoyo la comparación con otro concepto, que puede resultar más conocido por el estudiante, con la finalidad de distinguirlo. En esos casos, la operación comienza con el señalamiento de los puntos en común entre ambos objetos para destacar, sobre todo, aquellos que los distinguen y que hacen a la esencia del objeto de estudio en cuestión. Es importante, así, hacer hincapié en los criterios que sirven de eje para la comparación y para la distinción y en los recursos lingüísticos más empleados son aquellos de comparación, negación, concesión-restricción.

Por su parte, la explicación de un concepto teórico también es un ejemplo de las operaciones dicursivo-cognitivas que nos ocupan. En ese sentido, se debe señalar que en el campo de las ciencias sociales y humanas, la operación de explicación adopta características particulares debido a la naturaleza abstracta, teórica de sus objetos de conocimiento. En efecto, se trata de construcciones simbólicas que corresponden a contextos también abstractos. Son conceptos caracterizados por su naturaleza relativa y sistémica. Es decir que para esos conceptos no existe una definición universalmente aceptada, no tienen fronteras claras, límites definidos. Asimismo, esos conceptos se caracterizan por formar parte de un sistema de categorías relacionadas jerárquicamente, lo que implica que los razonamientos sustentados en este tipo de conceptos se basan en el establecimiento de relaciones y diferencias entre los distintos conceptos del sistema. Así entonces, la explicación de ese tipo de conceptos exige, en general, por un lado, poner de manifiesto su inscripción en el marco de una perspectiva disciplinar y, más específicamente, de una teoría dentro de esa perspectiva. Y, por otra parte, esa operación supone la explicitación de las conexiones existentes entre los distintos elementos que conforman el razonamiento.

Como último ejemplo, mencionaremos la operación, también muy frecuente en el campo de las ciencias humanas, que consiste en la exposición de distintas perspectivas sobre un mismo objeto de conocimiento, con el propósito de tomar postura ante el tema abordado desarrollando y defendiendo uno de los enfoques presentados. Efectivamente, en muchos textos primarios de ese campo disciplinar, la caracterización de la postura asumida por el autor no se presenta de manera aislada, sino que se construye en el marco de un debate con otras posiciones 
-vigentes en la comunidad disciplinar- de las cuales procura distinguirse. En esos casos, la operación de diferenciación se plantea como el complemento necesario de la caracterización ya que los rasgos definitorios de la postura son adjudicados gracias a su comparación y distinción con los otros enfoques expuestos.

\subsection{El segundo lineamiento}

El segundo lineamiento refiere tanto a la selección de los objetos de las prácticas - textos que se proponen para leer y escribir - como a las modalidades de lectura y escritura que favorecerían la articulación propuesta en la primera orientación.

\subsubsection{Los objetos de las prácticas}

Así, en cuanto a los textos que se seleccionan como material de lectura en la universidad, es necesario señalar su inscripción en determinados géneros discursivos cuyo propósito es construir y transmitir conocimientos. En ese sentido, la perspectiva sociocultural destaca el rol que desempeñan esos géneros en el aprendizaje de las disciplinas. En efecto, siguiendo a Cassany y Morales (2008) es dado reconocer que en ellos se elabora el conocimiento de cada campo y se construye la identidad de los miembros de la comunidad, escritores y lectores. Ahora bien, en función de favorecer - por medio de dispositivos concretos - el aprendizaje de esos modos propios de elaborar y transmitir el conocimiento por parte de los estudiantes en vistas a su inserción en la comunidad disciplinar, entendemos que es imprescindible aludir a las formas que adquieren las diferentes verbalizaciones del saber a las que los ellos se enfrentan. Así, en las bibliografías de las diferentes asignaturas se seleccionan "textos primarios" - textos fundadores, dirigidos a la comunidad de científicos y académicos - y "textos secundarios", en los cuales el saber de referencia ha sufrido modificaciones a los fines de la enseñanza y el aprendizaje. Como ejemplo de los primeros, podemos mencionar los artículos científicos, la tesis, los capítulos de libros teóricos; y entre los secundarios, situamos a las fichas de cátedra y los manuales. Esa distinción entre textos primarios y secundarios resulta productiva pues nos permite afirmar la necesidad de incluir en las bibliografías no sólo textos secundarios - cuyo acceso 
se presume más sencillo para los estudiantes - sino también textos fundadores. En ese sentido, en investigaciones anteriores hemos podido concluir que

considerando que el proceso de aprendizaje del conocimiento en el contexto universitario implica no sólo la apropiación del contenido conceptual sino también de las formas en que ese saber se construye, y que estas lógicas se ponen de manifiesto en los textos fundadores, no resultaría suficiente circunscribirse al tratamiento del texto secundario, aún si éste no altera el saber de referencia (...) De este modo, junto con el contenido en sentido estricto, lo que está en juego es la capacidad de apropiarse de razonamientos y de formas de argumentación propias de una disciplina, y el texto primario se constituye en el punto de apoyo a partir del cual los estudiantes pueden entrar progresivamente "en un mundo de referencias, de maneras de decir y de razonar" (LAHIRE citado por MILLET, 1999 , p. 5) específicas del campo en el que se están formando (DORRONZORO LUCHETTI, 2013, p. 1148-9).

Dado entonces que la selección de los materiales debería estar guiada por el propósito de favorecer la articulación entre pensamiento y lenguaje a través del abordaje de las operaciones discursivo-cognitivas por medio de las cuales se construye el conocimiento, y que es en los textos primarios donde se pone de manifiesto la lógica de esa construcción, resulta imprescindible, para nosotras, poner a los estudiantes en contacto con esos textos y diseñar intervenciones precisas para su estudio.

Por otro lado, y en lo atinente a los textos que se propone escribir para propiciar esa articulación, diremos que se trata de textos cuya escritura está íntimamente relacionada con la lectura, es decir, producciones que se elaboran a partir de la lectura y para volver a ella, en función de la construcción del conocimiento. Nuestra propuesta se aleja entonces de las prácticas en las que se escribe exclusivamente al finalizar el proceso de comprensión, circunscriptas a las instancias de evaluación como mero hecho comprobatorio y que, por consiguiente, se limitan exclusivamente a la verificación de lo aprendido por los estudiantes y están centradas en "decir el conocimiento" (BEREITER; SCARDAMALIA, 1992). En ese sentido, nos guía la intención de superar una tendencia habitual en la universidad, donde, según Solé et al. (2006), prevalecen las tareas más bien simples que implican por lo general la lectura y consulta de una única fuente y la resolución de exámenes que exigen la recuperación y el reconocimiento de la información, más que promover su elaboración. Se trataría, en consecuencia, 
de tareas que se consideran potenciadoras en mayor medida del aprendizaje reproductivo que del constructivo. En esa misma línea, Casco (2014) señala que en esos textos la escritura reproduce

de manera errática materiales (para ellos [los estudiantes])) opacos a los que se ha reducido mediante una simple operación de omisión. La escritura se convierte así en un mero acto de manipulación de palabras ajenas que, como objetos vacíos, se trasladan de un lado a otro y se yuxtaponen en subproductos textuales cercanos al collage (...) Se "cumple" con la tarea sin que se produzca aprendizaje y se activa una conducta que, de volverse rutinaria, pondrá en peligro las posibilidades futuras de hacer evolucionar los propios conocimientos en otros contextos y sin guía. (CASCO, 2014, p. 246).

Por el contrario, entendemos que es necesario que los textos que se escriben tiendan a "transformar el conocimiento" (BEREITER; SCARDAMALIA, 1992). Planteamos, entonces, la producción progresiva de escritos de diversa naturaleza y complejidad - notas marginales, cuadros, resúmenes, informes, entre otros elegidos cuidadosamente teniendo en cuenta las particularidades de los materiales con cuya lectura se articulan y, específicamente, con la lógica de construcción del conocimiento - la operación discursivo-cognitiva - involucrada en la elaboración del saber puesto en texto. En ese sentido, coincidimos con Cassany y Morales (2008) cuando plantean que

los estudiantes que inician una carrera universitaria se enfrentan al aprendizaje de las prácticas letradas nuevas, las propias de la disciplina que empiezan a estudiar, sea ingeniería, derecho, periodismo o cualquier otra. Estas prácticas son complejas, utilizan discursos altamente especializados y exigen saber construir significados de modo específico, de acuerdo con una tradición preestablecida." (CASSANY y MORALES, 2008, p.3).

Esa constatación exige, para nosotras, considerar el diseño de intervenciones didácticas enfocadas a la enseñanza de esas nuevas prácticas letradas, que comprenderán instancias de revisión y reformulación. De ese modo, se irá ampliando sucesivamente el repertorio de géneros académicos cuya apropiación, a la vez que promueve el proceso de aprendizaje disciplinar, permite a los estudiantes desplegar una voz propia y autónoma e incluirse, por esta vía, en la comunidad de referencia. 


\subsubsection{Las modalidades de lectura y escritura}

Pasemos, ahora, al abordaje del otro aspecto que compone este segundo lineamiento: las modalidades de lectura y escritura. Así entonces, en cuanto a las primeras, es posible describir diferentes modos de leer, definidos en relación con sus objetivos. En nuestro caso, y considerando nuestros propósitos, procuraremos superar las modalidades de corte contenidista, tales como la "lectura extractiva o la procesual" (MARIN, 2007; HALL, 2007), que persiguen como finalidad principal la recuperación de la información del texto, focalizadas exclusivamente en lo que los textos dicen y que soslayan los modos de decir con los cuales se construye el conocimiento. Por el contrario, y siguiendo a Millet (1999), privilegiaremos la "lectura crítica", modalidad empleada cuando se debe construir un conocimiento a partir de textos donde el contenido no se presenta de manera unívoca. La clave de la lectura no residirá en la identificación y la extracción de informaciones, cuyo único objetivo es la búsqueda de un elemento de conocimiento preciso. $\mathrm{Al}$ contrario, para nosotras se trata de desplegar

una práctica de lectura sostenida (relativamente larga y lineal), hermenéutica y crítica, que despeja la información textual, interpreta, y que, armada de la escritura, se efectúa lápiz en mano (...) Tomando nota de los pasajes importantes de un texto, parafraseando las ideas-fuerza de una argumentación, revelando los términos centrales del lenguaje conceptual de un autor, fijando ciertas citas sintéticas, comentando por escrito los puntos de acuerdo o de desacuerdo, estos estudiantes realizan una serie de procedimientos intelectuales que sustentan la realización de una lectura 'activa' (...) una lectura que recorta, selecciona, enfatiza, desata, explicita, comenta el texto a apropiarse"33 (MILLET, 1999, p. 6).

La apropiación de los textos fundadores exige, según el autor, una lectura activa que implica una relación de fuerzas entre el lector y el texto en la cual el lector no se somete a él, sino que intenta hacerlo suyo, dominando sus conceptos y utilizando la escritura para escribir "su" lectura - su interpretación del texto.

Por su parte, y en cuanto a las modalidades de escritura, "la modalidad interpretativa" (TOLCHINSKY, 1993) se revela, para nosotras, como la más apropiada para desarrollar la articulación entre pensamiento y lenguaje. Dicha 
práctica se manifiesta en producciones orientadas a reconstruir y explicitar el sentido del texto de estudio a partir de la interpretación - y no de la copia fiel que hace el estudiante, quien buscará dar, en su escrito, una forma inteligible al contenido del texto fuente para sus interlocutores. En ellas, el autor se distancia del texto fuente y de su situación de producción original, distinta de la situación de producción del nuevo escrito. El contenido temático sobre el que se escribe se encuentra estrechamente imbricado con el espacio retórico, concerniente al texto y a sus objetivos, y se consideran para su elaboración aquellas operaciones discursivo-cognitivas necesarias para llevar a cabo tal o cual objetivo, lo que exige la reelaboración de dichos contenidos y su transformación para adecuarse a la situación. Se puede decir, entonces, que esas producciones son el resultado de un proceso complejo de solución de problemas y que favorecen la adquisición y la integración de nuevos conocimientos, la reorganización, incluso la transformación del contenido, en la cual se pone de manifiesto la vinculación entre contenido disciplinar y lenguaje (TOLCHINSKY, 1993).

Desde nuestra perspectiva, esa modalidad interpretativa permite poner en relieve el valor epistémico de la escritura, es decir, su potencialidad en relación con la generación del conocimiento y su difusión. En ese sentido, “concretar el potencial epistémico a través de los textos supone involucrarse en propuestas que exijan ir más allá de reconocer, reproducir y fijar informaciones, o usarlas en contextos y condiciones muy parecidos a aquellos en que se aprendió" (SOLÉ et al., 2006, p. 161). Ahora bien, ¿qué actividades de escritura presentan un mayor potencial epistémico, y, por lo tanto, resultan necesarias de ser incluidas en el dispositivo que nos ocupa? Siguiendo a Solé et al. (2006), se trataría de "tareas híbridas", propuestas que involucran tanto la lectura como la escritura, en las que los estudiantes asumen el doble rol de lector y autor, tanto de los textos de la bibliografía como de sus propias producciones. Así,

este desdoblamiento, que obliga a descentrarse y a tomar en cuenta diversas perspectivas, así como el hecho de que las operaciones cognitivas implicadas en la lectura y la escritura, sin ser idénticas ni confundirse, guarden estrechas relaciones entre sí, explicaría por qué la combinación de ambas en la resolución de determinadas tareas potencia el pensamiento y el aprendizaje" (FITZGERALD; SHANAN, 2000, citados por SOLÉ, 2006).

Ejemplo de esas tareas híbridas son las respuestas a preguntas sobre un texto 
y la producción de diversos géneros - cuadros, resúmenes, síntesis, ensayos, informes - a partir de una o más fuentes, complementarias o contrastadas. En este sentido, y considerando que el dispositivo que proponemos busca propiciar la articulación entre contenido disciplinar y lenguaje en función del trabajo con las operaciones cognitivas en las que se materializa dicha articulación, es fundamental pensar cuáles de las producciones que se propongan resultarán más apropiadas, es decir, cuáles permitirán enseñar a reconstruir el proceso de construcción del conocimiento que se pone en juego en los diferentes textos y cuya aprehensión por parte del estudiante permitirá la conceptualización buscada.

Considerando, entonces, que la orientación que acabamos de desarrollar atiende tanto a la elección de los objetos de las prácticas como a las modalidades de lectura y escritura que favorecerían la articulación propuesta en el primer lineamiento, intentaremos plantear un ejemplo.

Así, el dispositivo sustentado en las principios expuestos precedentemente tendría el propósito, a través de la enseñanza de la modalidad de lectura crítica, de promover la apropiación no sólo de un contenido disciplinar específico, sino también del modo en que ese conocimiento es construido. Con tal intención, se podría proponer la lectura de dos textos diferentes: un texto primario, con dominante argumentativa y un texto secundario, explicativo, extraído de un manual. En el primero de ellos, la operación discursivo cognitiva que sustenta el razonamiento es la caracterización de diversas posturas respecto de un determinado objeto de estudio, para plantear y justificar el enfoque adoptado por los autores. En el texto secundario, la operación de base se limita a la exposición de las mismas posturas desarrolladas en el texto primario sin que se evidencie en este segundo escrito la asunción de una perspectiva particular por parte del autor. Así entonces, el dispositivo debería incluir intervenciones e instrucciones de lectura orientadas a propiciar la comprensión de las particularidades de las diferentes textualizaciones del saber que se presentan en los dos textos, a reconocer las diversas operaciones que se ponen en juego en cada uno de ellos, y a provocar la articulación entre ambos escritos. Se propondría así el abordaje sucesivo de los dos textos, iniciando el trabajo con la lectura del texto secundario, para que ésta se vuelva facilitadora de la comprensión de las posturas involucradas en el texto primario. Por su parte, el trabajo específico con el texto primario, andamiado por la tarea inicial realizada con el texto secundario, estaría centrado ya no en la caracterización per se de los diferentes enfoques - lo que limitaría el aprendizaje a la reproducción de los 
atributos de esas posturas - sino que avanzaría en la reconstrucción de la manera en que fue elaborado el conocimiento en ese texto fundador y, por esa vía, a concretar la articulación entre modos de pensar y modos de decir.

En ese mismo sentido, la lectura de los textos seleccionados se plantearía en articulación con prácticas de escritura instrumentales - tareas híbridas -, diferenciadas en función de las particularidades de los escritos elegidos. De ese modo, la toma de notas que acompañaría la lectura del texto secundario, podría revelarse como una práctica apropiada para una primera instancia en la que se busque la caracterización de las posturas presentes en ese texto. Esas primeras notas serían luego retomadas para promover la comprensión del texto primario, es decir, tendrían como finalidad acompañar la identificación de las características definitorias de cada una de las perspectivas desarrolladas en el texto primario, para luego distinguirlas entre ellas. En ese momento, se guiará a los estudiantes en la relectura de los textos que dé lugar, a su vez, a la revisión y completamiento de las notas, en un proceso recursivo en el que prime la interacción entre las prácticas lectivas y escriturarias.

Así entonces, para facilitar el establecimiento de relaciones y comparaciones, se propondrá que las notas tomadas se organicen en un cuadro comparativo. En esa actividad, las intervenciones y consignas estarían encaminadas a guiar a los estudiantes para el establecimiento de criterios que permitan caracterizar las diferentes perspectivas en juego. La escritura se presentará así no solo como un instrumento para decir lo comprendido, sino como una herramienta para la conceptualización de los rasgos definitorios de dichas perspectivas. Se trataría de una producción escrita destinada a superar la realización de una lista de atributos desarticulados, copiados literalmente del texto, sin que medie ninguna transformación. Al contrario, se propenderá a través de este escrito, a favorecer el establecimiento de relaciones, la comparación y la diferenciación a través del reordenamiento de las ideas que implicará la producción escrita.

\section{A modo de cierre}

El proceso de enseñanza aprendizaje en la universidad implica, para nosotras, ir más allá de las tradiciones ancladas en la mera transmisión del contenido disciplinar. Sin desconocer la centralidad de esa transmisión, en el contexto actual nuevas problemáticas vinculadas con las prácticas de lectura y escritura 
orientadas a la apropiación de los saberes disciplinares se hacen evidentes y nos interpelan. En ese marco, creemos, desde la perspectiva sociocultural, que aprender los conocimientos de cada disciplina es aprender a emplear los modos de discurso que le son propios y constitutivos. La tarea del docente, entonces, debería estar regida por el propósito de mostrar la inscripción del contenido disciplinar en el entramado de formas particulares de construir y expresar el conocimiento propias de ese campo del saber, es decir, en los modos específicos de relacionar los conceptos, de plantear los problemas y de representar la realidad.

Ahora bien, para que lo dicho no se vea limitado a una formulación de principios, creemos que resulta imprescindible que los mismos se actualicen en propuestas didácticas precisas. En este sentido, los lineamientos que hemos desarrollado desde la perspectiva sociocultural pretenden constituirse en sustentos para la elaboración de dispositivos didácticos, entendidos como recursos de mediación y acción. Esos recursos simbólicos, cuidadosamente planificados, estarán orientados a superar la dicotomía entre los planteos contenidistas y extractivos que centran la actividad en la transmisión-apropiación de los contenidos disciplinares, y aquellos que se focalizan exclusivamente en el tratamiento lingüístico del texto. Así, entendemos que no es en la escisión, sino en la articulación entre contenido y lenguaje donde se da la construcción del conocimiento. En efecto, si, como hemos planteado, el conocimiento disciplinar es un hecho discursivo, una construcción social llevada a cabo por medio de la comunicación instaurada por el lenguaje, resulta necesario desarrollar acciones - en ese caso, la elaboración de dispositivos contextualizados - que atiendan justamente a esa articulación. De otro modo, la didáctica negaría por alguna vía - por la del contenido o por la del lenguaje - a la epistemología, es decir, contradiría el modo mismo en que el conocimiento es construido, sus propias características constitutivas y definitorias. 


\title{
Didactic devices for the written language teaching and learning process at the university: some guidelines
}

\begin{abstract}
In this article, we will present a series of theoretical-methodological guidelines for the design of "didactic devices" for the teaching and learning of contents in subjects within the humanistic careers. These guidelines, developed within the framework of a Program of Accompaniment to teachers of the career of Education Sciences of the Universidad Nacional de Luján (Buenos Aires Province, Argentina), are in accordance with the Vygotskian sociocultural perspective. Therefore, they seek to show the link between the disciplinary contents and the forms of discourse through which they take place as well as the proper reasoning of each field of knowledge.
\end{abstract}

Keywords: Didactic devices. "Disciplinary knowledge”. Written language. University.

\section{Referencias}

BAUTIER, Elisabeth. Quand le discours pédagogique entrave la construction des usages littéraciés du langage. Pratiques. Université de Lorraine (France), n. 143/144, p. 11-26, déc. 2009.

BEREITER, Carl; SCARDAMALIA, Marlene. Dos modelos explicativos de los procesos de composición escrita. Infancia y Aprendizaje, Universidad Autónoma de Madrid: España, n. 58, p. 43-64, 1992.

CALSAMIGLIA, Helena. Decir la ciencia: Las prácticas divulgativas en el punto de mira. Revista Iberoamericana de Discurso y Sociedad, número monográfico, Barcelona (España), v. 2, n. 2, p. 3-8, jun. 2000.

CASCO, Miriam. Afiliación intelectual y escritura en la universidad: un estudio de caso sobre la perspectiva de los docentes. Bellaterra Journal of Teaching \& Learning Language \& Literature, España, v. 7, n. 2, p. 34-53, May-June 2014. CASSANY, Daniel; LÓPEZ FERRERO, Carmen; MARTÍ, Jaume. La transformación divulgativa de redes conceptuales científicas. Hipótesis, modelo y estrategia. Revista Iberoamericana de Discurso y Sociedad, número monográfico, Barcelona (España), v. 2, n. 2, p. 73-103, enero 2000. 
CASSANY, Daniel; MORALES, Oscar. Leer y escribir en la universidad: hacia la lectura y la escritura crítica de géneros científicos. Revista Memoralia, Universidad Nacional Experimental de los Llanos Ezequiel Zamora, Cojedes, (Venezuela), 2008.

CASTELLÓ, Monserrat. Aprender a escribir textos académicos: ¿Copistas, escribas, compiladores o escritores? In: POZO, Juan I.; PEREZ ECHEVERRIA, Ma. del Puy (Coord.). La psicología del aprendizaje universitario: de la adquisición de conocimientos a la formación en competências. Madrid: Morata, 2009. cap. 7, p. 120-133.

CUBERO PÉREZ, Rosario; CUBERO PÉREZ, Mercedes; SANTAMARÍA, Andrés; SAAVEDRA MACIAS, Javier; YUSSEF, Juan José. Aprendizaje y Psicología Histórico-Cultural. Aportaciones de una perspectiva social del aula. Investigación en la escuela, Universidad de Sevilla, p. 5-16, jun. 2007.

DELCAMBRE, Isabelle. Introduire des étudiants à la compréhension des discours universitaires. Première partie : quand les étudiants lisent pour comprendre. Recherches, Hergnies (France), n. 58, Lire et comprendre, p. 177-198, juin. 2013. DORRONZORO, Ma. Ignacia; LUCHETTI, Ma. Fabiana. Los textos en el ingreso a la universidad: análisis conceptual y discursivo. In: Jornadas Internacionales de Investigación y Prácticas en Didáctica de las Lenguas y las Literaturas, terceras, 2013, San Carlos de Bariloche. Terceras Jornadas Internacionales de Investigación y Prácticas en Didáctica de las lenguas y las literaturas. San Carlos de Bariloche: Ediciones GEISE, 2013.p. 1.133-1.152.

HALL, Beatriz. La comunicación científica en ámbitos académicos: otro enfoque. Hologramática, Facultad de Ciencias Sociales - UNLZ, año 4, v. 2, n. 7, p. 79105 , agosto 2007.

KOZULIN, Alex. Instrumentos psicológicos. La educación desde una perspectiva sociocultural. Madrid: Paidós, 2000.

LERNER, Delia; AISENBERG, Beatriz; ESPINOZA, Ana. La lectura y la escritura en la enseñanza de Ciencias Naturales y de Ciencias Sociales. Una investigación en didácticas específicas. Anuario de Investigaciones en Ciencias de la Educación. Buenos Aires, IICE - Facultad de Filosofía y Letras - UBA, p. 529-541, 2011.

MARIN, Marta. Lectura de textos de estudio, pensamiento narrativo y pensamiento conceptual. Hologramática, Facultad de Ciencias Sociales UNLZ, año 6, v. 4, n. 7, p. 61-80, agosto 2007. 
MILLET, Mathias. Économie des savoirs et pratiques de lecture. Une contribution à l'analyse des formes du travail intellectuel étudiant en médecine et en sociologie. Education et sociétés. Revue internationale de sociologie de l'éducation, Ecole Normale Supérieure de Lyon (France), n. 4, p. 57-74, enero 1999.

MORALES, María Alejandra; LENOIR, Yves; JEAN, Valérie. Dispositivos didácticos en la enseñanza primaria de Québec. Revista Iberoamericana de Evaluación Educativa, Barcelona (España), v. 5, n. 3, p. 115-132, 2012.

NUSSBAUM, Luci; TUSÓN, Amparo. El aula como espacio cultural y discursivo. Signos. Teoría y práctica de la educación, Universidad de Buenos Aires (Argentina), n. 17, p. 14-21, enero-marzo 1996.

OLSON, David. EI mundo sobre el papel. Barcelona: Gedisa Editorial, 1998.

RIVIÈRE, Ángel. La psicología de Vygotski. $5^{\text {ta. }}$ ed. Madrid: A. Machado Libros, 2002.

SOLÉ, Isabel; CASTELLS, Nuria; GRACIA, Marta; ESPINO, Sandra. Aprender psicología a través de los textos. Anuario de Psicología, Facultad de Psicología. Universidad de Barcelona (España), v. 37, n. 1-2, p. 157-176, abr.-sept. 2006.

TOLCHINSKY, Liliana. Aprendizaje del lenguaje escrito: procesos evolutivos e implicaciones didácticas. Barcelona: Anthropos, 1993.

VIGOTSKI, Lev. Pensamiento y linguaje: Teoría del desarrollo cultural de las funciones psíquicas. Buenos Aires: La Pléyade, 1977.

VIGOTSKI, Lev. El desarrollo de los procesos psicológicos superiores. Barcelona: Grupo Editor Grijalbo, 1978. 